\title{
A TWO-STRAIN EPIDEMIC MODEL WITH UNCERTAINTY IN THE INTERACTION
}

\author{
M. G. ROBERTS
}

(Received 30 July, 2012; revised 15 October, 2012; first published online 21 February, 2013)

\begin{abstract}
Annual epidemics of influenza A typically involve two subtypes, with a degree of cross-immunity. We present a model of an epidemic of two interacting viruses, where the degree of cross-immunity may be unknown. We treat the unknown as a second independent variable, and expand the dependent variables in orthogonal functions of this variable. The resulting set of differential equations is solved numerically. We show that if the population is initially more susceptible to one variant, if that variant invades earlier, or if it has a higher basic reproduction number than the other variant, then its dynamics are largely unaffected by cross-immunity. In contrast, the dynamics of the other variant may be considerably restricted.
\end{abstract}

2010 Mathematics subject classification: 92D30.

Keywords and phrases: epidemics, influenza, SIR model, immunity.

\section{Introduction}

Annual epidemics of influenza occur in all temperate regions of the world [5]. Each winter it is common for the epidemic to feature three variants of the virus: influenza A H1N1, influenza A H3N2, and influenza B. The relative proportions of each variant change from year to year [5], and are monitored by a national surveillance scheme in New Zealand [7]. The degree of cross-immunity between the influenza A subtypes has been a matter of speculation $[2,4,6]$, but is a critical determinant of the between-year dynamics of influenza [9].

Perhaps the best-known model for the spread of an epidemic is the so-called SIR model. The host population is of constant size, $N$ individuals, of which $S$ are susceptible to infection, $I$ are infectious, and $R$ are recovered and immune. The dynamics may be specified by two differential equations,

$$
\frac{d I}{d \tilde{t}}=\beta \frac{S I}{N}-\gamma I, \quad \frac{d R}{d \tilde{t}}=\gamma I,
$$

Institute of Natural \& Mathematical Sciences, New Zealand Institute for Advanced Study and Infectious Disease Research Centre, Massey University, Private Bag 102 904, North Shore Mail Centre, Auckland, New Zealand; e-mail: m.g.roberts@massey.ac.nz.

(C) Australian Mathematical Society 2013, Serial-fee code 1446-1811/2013\$16.00 
with $N=S+I+R$ and $\tilde{t}$ signifying time. The parameter $\beta$ is the contact rate between individuals multiplied by the probability of transmission given contact between a susceptible and an infectious individual, and $\gamma$ is the rate at which individuals recover from being infectious. The equations may be nondimensionalized by setting $t=\gamma \tilde{t}$ and substituting $I=y N$ and $I+R=z N$ to obtain

$$
\frac{d y}{d t}=\mathcal{R}_{0} y(1-z)-y, \quad \frac{d z}{d t}=\mathcal{R}_{0} y(1-z),
$$

where $\mathcal{R}_{0}=\beta / \gamma$ is the basic reproduction number [3, 8].

As immunity is postulated to be "short lived", we introduce a parameter $q$ where $q=0$ corresponds to no cross-immunity and $q=1$ implies that exposure to one virus variant in this season confers total immunity to the alternate variant for the rest of the season. Using the superscripts $s$ and $r$ to denote the two virus variants, the proportion of the population that was initially immune to variant $s$ and has since been exposed to either variant is $u^{s}$, where

$$
\frac{d u^{s}}{d t}=\left(\mathcal{R}_{0}^{s} y^{s}+\mathcal{R}_{0}^{r} y^{r}\right)\left(1-u^{s}\right), \quad u^{s}(0)=z^{s}(0) .
$$

Hence

$$
-\int_{0}^{t}\left(\mathcal{R}_{0}^{s} y^{s}(\tau)+\mathcal{R}_{0}^{r} y^{r}(\tau)\right) d \tau=\log \left(\frac{1-u^{s}(t)}{1-u^{s}(0)}\right)
$$

and

$$
1-u^{s}(t)=\frac{\left(1-z^{s}(t)\right)\left(1-z^{r}(t)\right)}{1-z^{r}(0)} .
$$

The effective susceptibility of the population to variant $s$ is then

$$
x^{s}=1-u^{s}+(1-q)\left(u^{s}-z^{s}\right)=(1-q)\left(1-z^{s}\right)+q\left(1-u^{s}\right) .
$$

Observe that, if there is complete cross-immunity then $q=1$ and $x^{s}=1-u^{s}$, whereas if there is no cross-immunity then $q=0$ and $x^{s}=1-z^{s}$.

\section{The model}

We now analyse the two-variant model

$$
\frac{d y^{s}}{d t}=\mathcal{R}_{0}^{s} x^{s} y^{s}-y^{s}, \quad \frac{d z^{s}}{d t}=\mathcal{R}_{0}^{s} y^{s}\left(1-z^{s}\right),
$$

for $s=1,2$, with

$$
\frac{x^{s}(t)}{1-z^{s}(t)}=1-q+q \frac{1-z^{r}(t)}{1-z^{r}(0)}
$$

when $s=1, r=2$, and vice versa. As $q$ is unknown, we treat it as a random variable and expand

$$
y^{s}(t, q)=\sum_{i=1}^{\infty} y_{i}^{s}(t) \psi_{i}(q), \quad z^{s}(t, q)=\sum_{i=1}^{\infty} z_{i}^{s}(t) \psi_{i}(q)
$$


where $\left\{\psi_{i}\right\}$ is a set of functions defined on $[0,1]$ such that the expectation $\mathbb{E}\left(\psi_{i} \psi_{j}\right)=0$ if $i \neq j$. Substituting the expansions (2.3) in equation (2.2),

$$
\begin{aligned}
x^{s}(t, q)= & (1-q)\left(1-z^{s}(t, q)\right)+\frac{q}{1-z^{r}(0)}\left(1-z^{s}(t, q)\right)\left(1-z^{r}(t, q)\right) \\
=1 & +\frac{q z^{r}(0)}{1-z^{r}(0)}-\left(1+\frac{q z^{r}(0)}{1-z^{r}(0)}\right) \sum_{j=1}^{\infty} z_{j}^{s}(t) \psi_{j}(q) \\
& \quad-\frac{q z^{r}(0)}{1-z^{r}(0)} \sum_{k=1}^{\infty} z_{k}^{r}(t) \psi_{k}(q)+\frac{q z^{r}(0)}{1-z^{r}(0)} \sum_{j=1}^{\infty} \sum_{k=1}^{\infty} z_{j}^{s}(t) z_{k}^{r}(t) \psi_{j}(q) \psi_{k}(q) .
\end{aligned}
$$

Substituting (2.3) in the first equation in (2.1), multiplying by $\psi_{\ell}(q)$ and taking expectations over $q$,

$$
\begin{aligned}
\mathbb{E}\left(\psi_{\ell}^{2}\right) \dot{y}_{\ell}^{s}=\mathcal{R}_{0}^{s}\left\{\mathbb{E}\left(\psi_{\ell}^{2}\right) y_{\ell}^{s}+\frac{z^{r}(0)}{1-z^{r}(0)} \sum_{i=1}^{\infty} \mathbb{E}\left(q \psi_{i} \psi_{\ell}\right) y_{i}^{s}\right. \\
-\sum_{i=1}^{\infty} \sum_{j=1}^{\infty} \mathbb{E}\left(\psi_{i} \psi_{j} \psi_{\ell}\right) y_{i}^{s} z_{j}^{s}-\frac{z^{r}(0)}{1-z^{r}(0)} \sum_{i=1}^{\infty} \sum_{j=1}^{\infty} \mathbb{E}\left(q \psi_{i} \psi_{j} \psi_{\ell}\right) y_{i}^{s} z_{j}^{s} \\
-\frac{1}{1-z^{r}(0)} \sum_{i=1}^{\infty} \sum_{k=1}^{\infty} \mathbb{E}\left(q \psi_{i} \psi_{k} \psi_{\ell}\right) y_{i}^{s} z_{k}^{r} \\
\left.+\frac{1}{1-z^{r}(0)} \sum_{i=1}^{\infty} \sum_{j=1}^{\infty} \sum_{k=1}^{\infty} \mathbb{E}\left(q \psi_{i} \psi_{j} \psi_{k} \psi_{\ell}\right) y_{i}^{s} z_{j}^{s} z_{k}^{r}\right\}-\mathbb{E}\left(\psi_{\ell}^{2}\right) y_{\ell}^{s} .
\end{aligned}
$$

We approximate the infinite system by truncating the series (2.3) after $N$ terms to obtain

$$
\begin{aligned}
\dot{y}_{\ell}^{s}= & \mathcal{R}_{0}^{s}\left\{y_{\ell}^{s}+a^{r} \mathbf{c}_{\ell} \cdot \mathbf{y}^{s}-\mathbf{z}^{s} \cdot \mathbf{A}_{\ell} \mathbf{y}^{s}-a^{r} \mathbf{z}^{s} \cdot \mathbf{B}_{\ell} \mathbf{y}^{s}-b^{r} \mathbf{z}^{r} \cdot \mathbf{B}_{\ell} \mathbf{y}^{s}\right\} \\
& +b^{r} \mathcal{R}_{0}^{s} \sum_{k=1}^{N} \mathbf{z}^{s} \cdot \mathbf{C}_{k \ell} \mathbf{y}^{s} z_{k}^{r}-y_{\ell}^{s}
\end{aligned}
$$

for $\ell=1, \ldots, N$, with $\mathbf{y}^{s}=\left(y_{1}^{s}, y_{2}^{s}, \ldots, y_{N}^{s}\right)^{\prime}$ and $\mathbf{z}^{s}$ defined similarly (here the prime means transpose). The $N \times N$ matrices $\mathbf{A}_{\ell}, \mathbf{B}_{\ell}, \mathbf{C}_{k \ell}$ have components

$$
A_{\ell i j}=\frac{\mathbb{E}\left(\psi_{i} \psi_{j} \psi_{\ell}\right)}{\mathbb{E}\left(\psi_{\ell}^{2}\right)}, \quad B_{\ell i j}=\frac{\mathbb{E}\left(q \psi_{i} \psi_{j} \psi_{\ell}\right)}{\mathbb{E}\left(\psi_{\ell}^{2}\right)}, \quad C_{k \ell i j}=\frac{\mathbb{E}\left(q \psi_{i} \psi_{j} \psi_{k} \psi_{\ell}\right)}{\mathbb{E}\left(\psi_{\ell}^{2}\right)},
$$

respectively. The vector $\mathbf{c}_{\ell}$ has components $c_{\ell i}=\mathbb{E}\left(q \psi_{i} \psi_{\ell}\right) / \mathbb{E}\left(\psi_{\ell}^{2}\right)$ and the constants are defined by $a^{s}=z^{s}(0) /\left(1-z^{s}(0)\right)$ and $b^{s}=1 /\left(1-z^{s}(0)\right)$ for $s=1,2$. Using the same technique, the second equation in (2.1) becomes

$$
\dot{z}_{\ell}^{s}=\mathcal{R}_{0}^{s}\left(y_{\ell}^{s}-\mathbf{z}^{s} \cdot \mathbf{A}_{\ell} \mathbf{y}^{s}\right) .
$$


Taken together, (2.4) and (2.5) comprise a set of $4 N$ ordinary differential equations for $\ell=1, \ldots, N$, with $s, r=1,2$ and $r \neq s$. The initial conditions are $\mathbf{y}^{s}(0)=y^{s}(0) \mathbf{e}$ and $\mathbf{z}^{s}(0)=z^{s}(0) \mathbf{e}$, where $\mathbf{e}$ is a unit vector with components $e_{i}=0$ for $i=2, \ldots, N$.

If we assume $q$ to be uniformly distributed between zero and one, the appropriate basis $\left\{\psi_{i}\right\}$ is the set of shifted Legendre polynomials [1, p. 774]. We take $\psi_{i}(q)=$ $\bar{P}_{i-1}(q)$, defining $\psi_{1}(q)=1, \psi_{2}(q)=2 q-1$ and

$$
\psi_{i+2}(q)=\frac{2 i+1}{i+1}(2 q-1) \psi_{i+1}(q)-\frac{i}{i+1} \psi_{i}(q), \quad i=1,2, \ldots
$$

The orthogonality relationship is $\mathbb{E}\left(\psi_{i} \psi_{j}\right)=\delta_{i j} /(2 i-1)$, where $\delta_{i j}=1$ if $i=j$ and $\delta_{i j}=0$ otherwise.

Equations (2.4) and (2.5) were solved numerically using MATLAB to explore the effect of the degree of cross-immunity (the value of $q$ ) on the dynamics of two-variant epidemics when the initial proportions in the population susceptible to each variant are different $\left(z^{1}(0)<z^{2}(0)\right)$, when the initial proportions in the population infectious with each variant are different $\left(y^{1}(0)>y^{2}(0)\right)$, when the basic reproduction numbers of each variant are different $\left(\mathcal{R}_{0}^{1}>\mathcal{R}_{0}^{2}\right)$, and a combination of the last two scenarios. The results are presented in Figures $1-3$.

\section{Results}

In Figure 1(a) and (b) it can be seen that if just $10 \%$ of the population is initially immune to variant two, and the whole population initially susceptible to variant one, then the epidemic of variant one is largely unaffected by the presence of variant two. However, and not surprisingly, the value of $q$ has a large effect on the epidemic of variant two. Similar patterns are observed when the initial proportion of the population infected with variant one is five times the proportion infected with variant two (Figure 1(c) and (d)), and when the basic reproduction number of variant one is bigger than that of variant two (Figure 1(e) and (f)). In all cases, the variant that dominates the initial part of the epidemic establishes a degree of cross-immunity in the population that may substantially reduce the size of the epidemic of the other variant.

In Figure 2, a scenario where each virus variant has a different advantage is illustrated, with $\mathcal{R}_{0}^{1}>\mathcal{R}_{0}^{2}$ and $y^{1}(0)>y^{2}(0)$. It is seen that the epidemics of both variants peak at approximately the same time, but that variant one infects a greater proportion of the population than variant two.

The results from all four scenarios are summarized in Figure 3. For the three scenarios where virus variant one has a clear advantage (Figure 3(a), (b) and (c)), the proportion of the population infected throughout the epidemic only reduces slightly from $z^{1}(\infty) \simeq 0.8$ when $q=0$ to a value $z^{1}(\infty)>0.7$ when $q=1$. In contrast, the proportion infected with variant two during the epidemic decreases substantially as $q$ is increased from zero to one. For the fourth scenario (Figure 3(d)), where variant two has a lower basic reproduction number but a higher initial proportion infected, the proportion of the population infected in the epidemic by each variant is reduced by less than half as $q$ is increased from zero to one. 
(a)

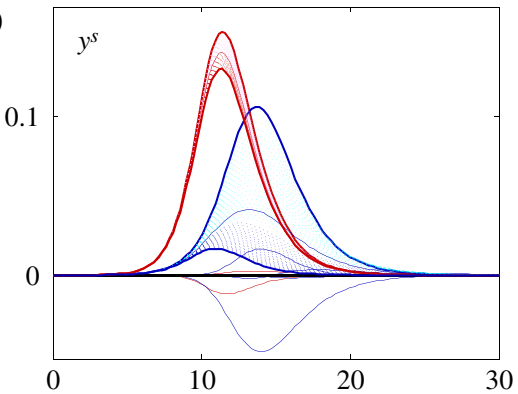

(c)

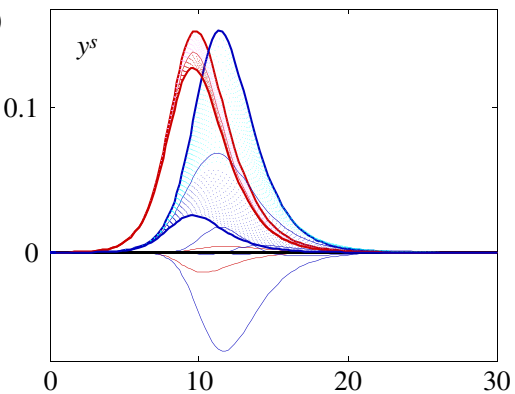

(e)

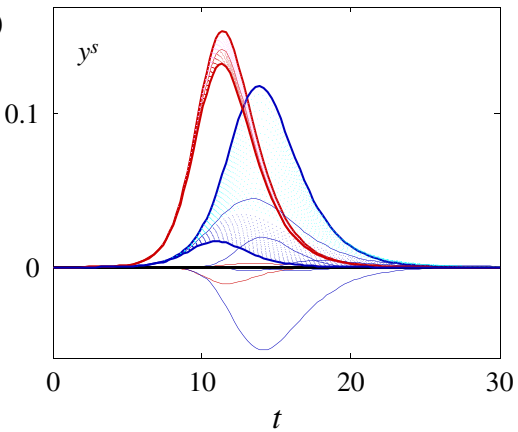

(b)

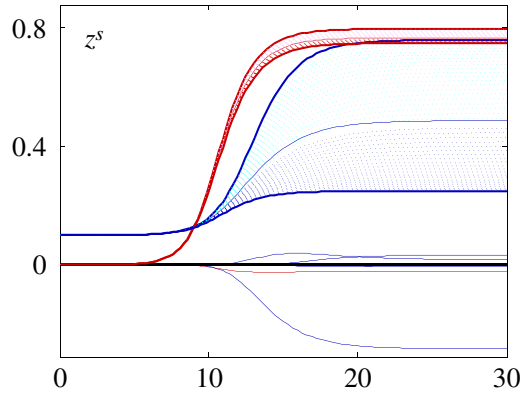

(d)

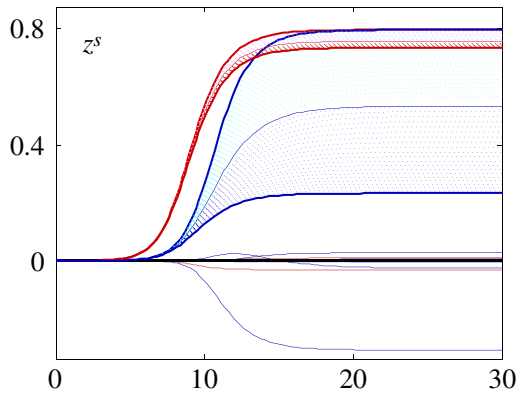

(f)

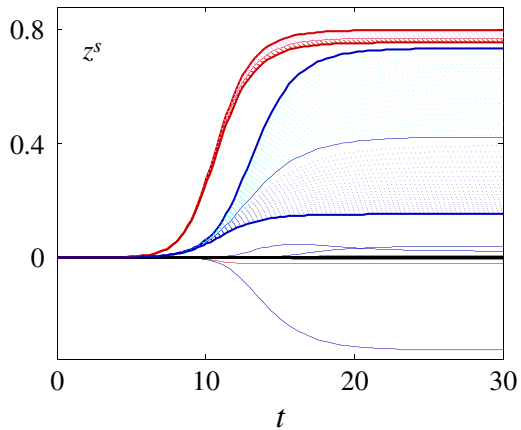

Figure 1. Variation of (left: a,c,e) the proportion of the population infected and (right: b,d,f) the proportion immune with time. The solid red lines are the extreme values (left) $y^{1}(t, 0), y^{1}(t, 1)$ and (right) $z^{1}(t, 0), z^{1}(t, 1)$; the solid blue lines are (left) $y^{2}(t, 0), y^{2}(t, 1)$ and (right) $z^{2}(t, 0), z^{2}(t, 1)$. Cyan and magenta shading shows $y^{s}(t, q), z^{s}(t, q)$ for $0<q<0.5$ and $s=1,2$; blue and red shading shows $y^{s}(t, q), z^{s}(t, q), 0.5<q<1$. The thin lines are solutions $y_{i}^{s}(t), z_{i}^{s}(t)$. Parameter values are $\mathcal{R}_{0}^{1}=\mathcal{R}_{0}^{2}=2$, $y^{1}(0)=y^{2}(0)=10^{-5}, z^{1}(0)=z^{2}(0)=0$ unless otherwise stated: (a,b) $z^{2}(0)=0.1,(\mathrm{c}, \mathrm{d}) y^{1}(0)=5 \times 10^{-5}$, (e,f) $\mathcal{R}_{0}^{2}=1.8$. (Colour available online.)

\section{Conclusion}

We have presented a method whereby the interaction between two viruses in an epidemic may be investigated. This could have been achieved by repeatedly solving the model equations for different values of the cross-immunity $q$, but instead we have 
(a)

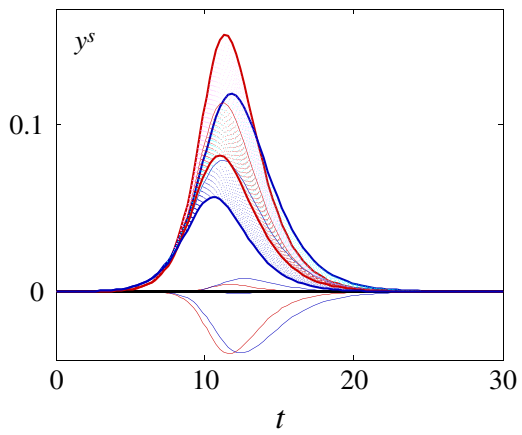

(b)

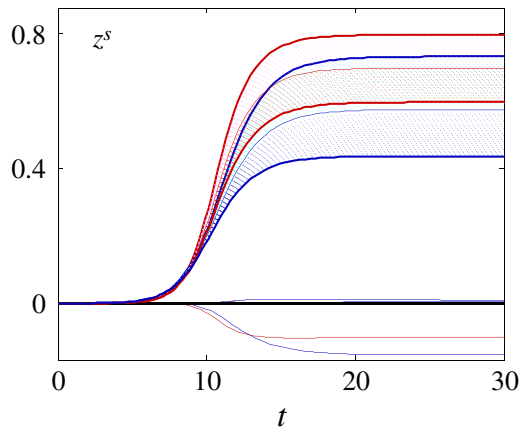

FigURE 2. Variation of (a) the proportion of the population infected and (b) the proportion immune with time. The solid red lines are the extreme values (a) $y^{1}(t, 0), y^{1}(t, 1)$ and (b) $z^{1}(t, 0), z^{1}(t, 1)$; the solid blue lines are (a) $y^{2}(t, 0), y^{2}(t, 1)$ and (b) $z^{2}(t, 0), z^{2}(t, 1)$. Cyan and magenta shading shows $y^{s}(t, q), z^{s}(t, q)$ for $0<q<0.5$ and $s=1,2$; blue and red shading shows $y^{s}(t, q), z^{s}(t, q), 0.5<q<1$. The thin lines are solutions $y_{i}^{s}(t), z_{i}^{s}(t)$. Parameter values: $\mathcal{R}_{0}^{1}=2, \mathcal{R}_{0}^{2}=1.8, y^{1}(0)=10^{-5}, y^{2}(0)=5 \times 10^{-5}, z^{1}(0)=z^{2}(0)=0$. (Colour available online.)

(a)

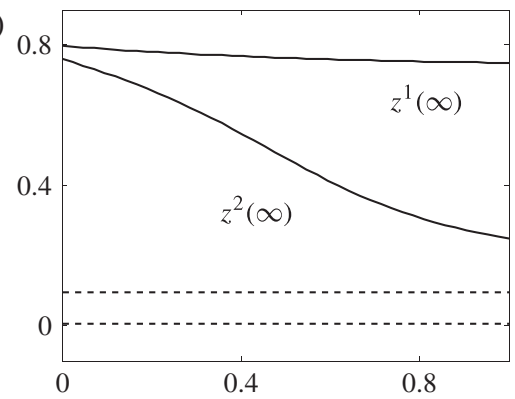

(c)

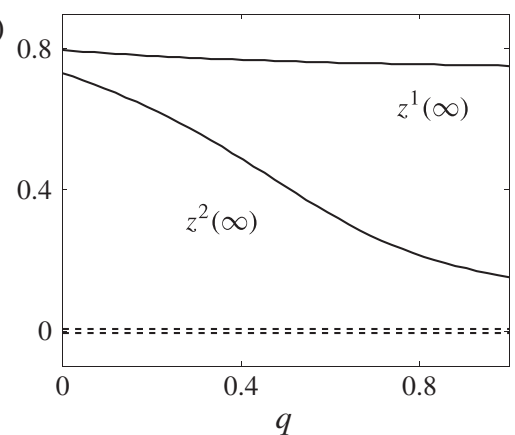

(b)

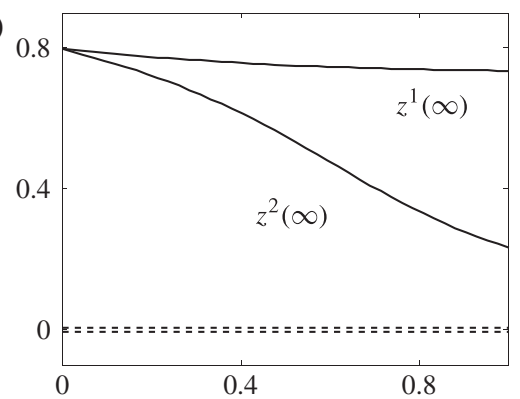

(d)

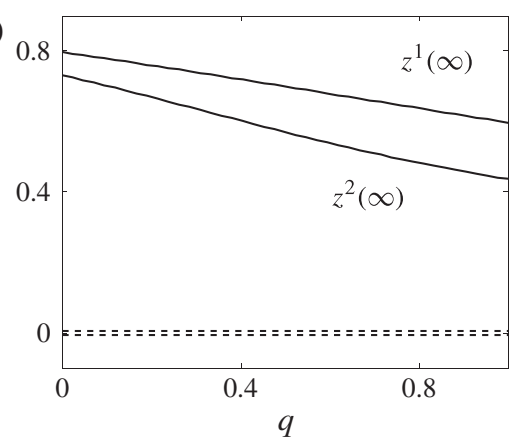

FIGURE 3. Variation of the proportion of the population immune to virus variant one, $z^{1}(\infty)$, and virus variant two, $z^{2}(\infty)$, at the end of an epidemic, as functions of the degree of cross-immunity $q$. Initial values are shown as horizontal broken lines. Parameter values are $\mathcal{R}_{0}^{1}=\mathcal{R}_{0}^{2}=2, y^{1}(0)=y^{2}(0)=10^{-5}$, $z^{1}(0)=z^{2}(0)=0$ unless otherwise stated: (a) $z^{2}(0)=0.1$; (b) $y^{2}(0)=5 \times 10^{-5}$; (c) $\mathcal{R}_{0}^{2}=1.8$; (d) $\mathcal{R}_{0}^{2}=1.8$ and $y^{2}(0)=5 \times 10^{-5}$. 
treated $q$ as a second independent variable. A set of ordinary differential equations was then constructed by expanding the state variables in orthogonal polynomials in $q$. This method has the advantage over repeated individual solutions of the equations in that it presents a comprehensive illustration of the effect of cross-immunity on the dynamics of the epidemic as the result of one calculation. We have previously used this method to investigate the sensitivity of the epidemic curve to uncertainty in the estimate of the basic reproduction number, where a single virus caused an epidemic [10]. There, and here, the method is fully deterministic once parameters have been assigned. Despite implications to the contrary $[11,12]$, the method is not stochastic and does not involve chaos.

Our results show that even a small degree of cross-immunity can give considerable advantage to a virus variant. We showed that where the population was initially more susceptible to variant one, the presence of cross-immunity had minimal effect on the dynamics of that variant. In contrast, variant two could be considerably suppressed: see Figure 1(a) and (b). A similar situation resulted when the initial prevalence of variant one was assumed to be five times that for variant two (Figure 1(c) and (d)). This is equivalent to infection of the population with variant one being initiated at an earlier time. A second similar result was obtained when the basic reproduction number for variant one was higher than that for variant two (Figure 1(e) and (f)). An interesting topic for further study is the detailed nature of the trade-off between a higher basic reproduction number, and an earlier introduction of infection to the population, as illustrated in Figure 2. It has been observed that seasonal epidemics of influenza A involve two subtypes, $\mathrm{H} 1 \mathrm{~N} 1$ and $\mathrm{H} 3 \mathrm{~N} 2$, but the predominant subtype is different in different years and seemingly unpredictable. This analysis has shown how even small differences in population susceptibility, or in the timing or infectivity of the virus, when coupled with cross-immunity, can make a big difference to the selection of a dominant virus variant.

\section{Acknowledgements}

This work was carried out while the author was visiting the Institute for Mathematical Sciences, National University of Singapore, in 2011. The visit was supported by the Institute. The author acknowledges financial support by the Marsden Fund under contract MAU0809. The author would like to thank Carlo Laing for pointing out the book by Xiu [11].

\section{References}

[1] M. Abramowitz and I. A. Stegun (eds), Handbook of mathematical functions: with formulas, graphs and mathematical tables (Dover Publications, New York, 1972).

[2] V. Andreasen, "Dynamics of annual influenza A epidemics with immuno-selection", J. Math. Biol. 46 (2003) 504-536; doi:10.1007/s00285-002-0186-2.

[3] O. Diekmann and J. A. P. Heesterbeck, Mathematical epidemiology of infectious diseases: model building, analysis and interpretation (John Wiley \& Sons, Chichester, 2000). 
[4] N. M. Ferguson, A. P. Galvani and R. M. Bush, "Ecological and immunological determinants of influenza evolution", Nature 422 (2003) 428-433; doi:10.1038/nature01509.

[5] B. S. Finkelman, C. Viboud, K. Koelle, M. J. Ferrari, N. Bharti and B. T. Grenfell, "Global patterns in seasonal activity of influenza A/H3N2, A/H1N1, and B from 1997 to 2005: viral coexistence and latitudinal gradients”, PLOS ONE 2 (2007) e1296; doi:10.1371/journal.pone.0001296.

[6] K. Koelle, S. Cobey, B. T. Grenfell and M. Pascual, "Epochal evolution shapes the phylodynamics of interpandemic influenza A (H3N2) in humans", Science 314 (2006) 1898-1903; doi:10.1126/science.1132745.

[7] L. Lopez and Q. S. Huang, Influenza surveillance in New Zealand 2011 (Institute of Environmental Science and Research Ltd (ESR), Wellington, 2012), http://www.surv.esr.cri.nz/PDF_surveillance/Virology/FluAnnRpt/InfluenzaAnn2011.pdf.

[8] M. G. Roberts, "The pluses and minuses of $\mathcal{R}_{0}$ ", J. R. Soc. Interface 4 (2007) 949-961; doi:10.1098/rsif.2007.1031.

[9] M. G. Roberts, "The dynamics of seasonal influenza", in: Design and Analysis of Infectious Disease Studies (organized by M. Eichner, E. Halloran and P. D. O’Neill), Oberwolfach Rep. 6 (2009) 2673-2698; doi:10.4171/OWR/2009/48.

[10] M. G. Roberts, "Epidemic models with uncertainty in the reproduction number", J. Math. Biol., in press; doi:10.1007/s00285-012-0540-y.

[11] D. Xiu, Numerical methods for stochastic computations (Princeton University Press, Princeton, NJ, 2010).

[12] D. Xiu and G. E. Karniadakis, "The Wiener-Askey polynomial chaos for stochastic differential equations”, SIAM J. Sci. Comput. 24 (2002) 619-644; doi:10.1137/S1064827501387826. 